\title{
Tsafon
}

Revue d'études juives du Nord

$72 \mid 2016$

Juifs, Israéliens, dans la littérature française et israélienne

\section{La mort est mon métier de Robert Merle l'œuvre à contre-courant}

\section{Anne Wattel}

\section{OpenEdition}

\section{Journals}

Édition électronique

URL : https://journals.openedition.org/tsafon/356

DOI : $10.4000 /$ tsafon.356

ISSN : 2609-6420

Éditeur

Association Jean-Marie Delmaire

Édition imprimée

Date de publication : 1 décembre 2016

Pagination : 15-34

ISSN : $1149-6630$

Référence électronique

Anne Wattel, «La mort est mon métier de Robert Merle l'œuvre à contre-courant », Tsafon [En ligne], 72 | 2016, mis en ligne le 31 mai 2018, consulté le 23 juin 2021. URL : http://journals.openedition.org/ tsafon/356 ; DOl : https://doi.org/10.4000/tsafon.356 


\title{
La mort est mon métier de Robert Merle l'œuvre à contre-courant
}

\author{
Anne Wattel ${ }^{*}$
}

Dans sa préface à l'édition de 1972, Robert Merle qualifie La mort est mon métier ${ }^{1}$ de roman à contre-courant : " Quand je rédigeai La mort est mon métier, de 1950 à 1952, j'étais parfaitement conscient de ce que je faisais : j'écrivais un livre à contre-courant. Mieux même : mon livre n'était pas encore écrit qu'il était déjà démodé ». (MEMM I). Le participe passé «démodé », l'expression «à contre-courant» sont à assimiler au «retard» tel que le définit Benoît Denis, évoquant l'angoisse de l'écrivain engagé qui s'efforce de coller à son temps, son présent, mais qui ne peut synchroniser ce dernier avec le texte écrit car « le texte arrive toujours trop tard, il est toujours en décalage par rapport à l'événement dont il se saisit [...] $»^{2}$. La mort est mon métier est certes décalé, à contre-courant, et ce à plus d'un titre, temporellement, face à une doxa dominante, et par le choix de donner voix au bourreau.

Décalée, l'œuvre l'est au niveau temporel. Elle est publiée en 1952 et n'est plus conforme aux attentes de la période, pire, c'est le roman à contre-courant par excellence qui entend témoigner d'un passé que l'on veut, dans les années 1950, mettre entre parenthèses. L'on est entré dans une ère de l'amnésie. Annette Wieviorka a montré que dans l'immédiat

\footnotetext{
* Université Charles de Gaulle - Lille 3, EA 1061, ALITHILA - Analyses Littéraires et Histoire de la Langue.

${ }^{1}$ Notre étude portant sur La mort est mon métier, que nous aurons souvent l'occasion de citer, le titre sera abrégé comme suit et les paginations entre parenthèses renverront à l'édition suivante : Robert Merle, La mort est mon métier, [1952], Paris, Gallimard, coll. « Folio », 1972. Ex : (MEMM 12).

${ }^{2}$ Benoît Denis, Littérature et engagement de Pascal à Sartre, Paris, Seuil, coll. « Points Essais », 2000, pp. 38-39.
} 
après-guerre, en 1945, 1946, 1947, les publications de récits de survivants se sont multipliés, mais dès 1948 s'amorce le déclin: les lecteurs ne veulent plus des témoignages, les publications se tarissent faute de lectorat. Le vécu du survivant n'est pas tant indicible qu'inaudible, pour reprendre le titre d'un de ses articles ${ }^{3}$. De cette histoire-là, on ne veut plus. Le texte de Merle qui paraît en 1952 n'est pas seulement en retard, il est quasiment hors de propos. En outre, il est «politiquement incorrect», Merle publie La mort est mon métier en plein cœur du débat sur le réarmement de l'Allemagne, sur la CED (la Communauté Européenne de Défense), alors même qu'il s'agit de tourner la page, comme il le précise dans sa préface :

Immédiatement après 1945, on vit paraître en France nombre de témoignages bouleversants sur les camps de la mort outre-Rhin. Mais cette floraison fut brève. Le réarmement de l'Allemagne marqua le déclin, en Europe, de la littérature concentrationnaire. Les souvenirs de la maison des morts dérangeaient la politique de l'Occident : on les oublia. (MEMM I)

S'opposent donc bien ici l'écrit-témoignage dans son immédiateté criante, "immédiatement après », et l'air du temps, ère du déclin du témoignage, ère de 1'oubli, ou du moins du désir d'oubli à des fins politiques. Merle explique par là une des raisons de la réception plus que problématique de son roman dans les années cinquante et la reconnaissance de ce dernier a posteriori pour les générations nées après 1945, celles pour lesquelles La mort est mon métier est, comme il l'indique dans sa préface, « un livre d'histoire ». Mais on ne peut dénier à Merle, au vu de ses prises de position contre le réarmement de l'Allemagne et contre la CED, la volonté, envers et contre le courant des années cinquante, d'éviter l'oubli, quitte à déranger.

\section{2 : à contre-pied de la doxa dominante}

Le désaveu critique qui a pesé sur l'œuvre ne tient pas uniquement à ce retard, loin s'en faut, son roman heurte de plein fouet la doxa dominante concernant l'écriture sur et après Auschwitz. Il se heurte donc tout d'abord à des interdits majeurs, des tabous : l'interdit de la poésie après Auschwitz et le rejet d'une fictionnalisation des camps, le refus de tout discours qui n'émanerait pas d'un témoin survivant.

\footnotetext{
${ }^{3}$ Annette Wieviorka, « Indicible ou inaudible ? La déportation : premiers récits (19441947) », Pardès, 1989, 9-10, pp. 23-59.
} 
Comme l'indique Frédérik Detue ${ }^{4}$, auquel nous empruntons l'analyse qui suit, la césure historique d'Auschwitz s'est couplée d'une césure littéraire. L'après, c'est l'ère de « la littérature en suspens », pour reprendre l'expression d'Imre Kertész $z^{5}$. La célèbre phrase d'Adorno, " écrire de la poésie après Auschwitz est barbare », que l'on a analysé comme un interdit de la littérature après Auschwitz, doit en fait, de manière plus nuancée, s'entendre comme une dénonciation de la culture, une culture qui a échoué à transformer les hommes, qui n'a pu empêcher la barbarie et qui même l'a rendue possible. La restaurer après Auschwitz, voilà ce qui est barbare. Adorno appelait donc à une autre voie pour dire, une voie qui ne soit ni une résurgence de cette culture-là, ni un rejet de la culture. Par ailleurs, comme le stipule Dominique Viart, « ce ne sont pas tant les ouvrages consacrés aux camps qui sont regardés avec suspicion, mais ceux qui s'en approchent avec les moyens de la littérature $»^{6}$. Peut-on donner à l'indicible de la barbarie, du génocide un corps romanesque? La transcription de l'histoire, sa refiguration romanesque, pour reprendre les termes d'Emmanuel Bouju ${ }^{7}$, la relecture/retranscription subjective des faits ne sont-elles pas des trahisons? Le détour par la fiction, parce qu'il est détour justement, relève d'un interdit, interdit qu'affirme explicitement Élie Wiesel: " Auschwitz nie toute littérature comme il nie tous les systèmes ${ }^{8}$. Jorge Semprun reviendra sur la question de manière plus nuancée. Dans L'écriture ou la vie, il évoque la nécessité de l'artifice pour donner de la voix, pour que l'inouï puisse être ouï et atteigne son objectif, à savoir la transmission de l'expérience : « [...] raconter bien, ça veut dire de façon à être entendus. On n'y parviendra pas sans un peu d'artifice $»^{9}$. Cet artifice, comme il le précise, est celui d'un " récit maîtrisé ». Ce qui est donc en jeu ici, n'est pas tant s'il faut ou pas dire, mais comment dire.

Dès la parution de l'œuvre de Robert Merle, Jean Cayrol, dans la revue Esprit, d'avril $1953^{10}$, s'insurge contre cette « nouvelle matière

\footnotetext{
${ }^{4}$ Frédérik Detue, «Quand écrire, c'est blesser (les lecteurs) : témoignages des camps et communauté négative », Frédérik Detue et Christine Servais (dir.), La lecture littéraire et l'utopie d'une communauté, Études littéraires, vol. 41, nº 2, 2010, pp. 59-79.

${ }^{5}$ Imre Kertész, cité dans Catherine Coquio, La littérature en suspens. Écritures de la Shoah : le témoignage et les œuvres, Paris, L'Arachnéen, 2015.

${ }^{6}$ Dominique Viart, « Le scrupule du roman », Vacarme, n ${ }^{\circ} 54$, janvier 2011, pp. 26-28.

${ }^{7}$ Emmanuel Bouju, La transcription de l'histoire (essai sur le roman européen de la fin $d u X X^{e}$ siècle), Rennes, Interférences, Presses universitaires de Rennes, 2006.

${ }^{8}$ Élie Wiesel, Un juif aujourd'hui, Paris, Seuil, 1977, p. 191.

9 Jorge Semprun, L'écriture ou la vie, Paris, Gallimard, coll. « Folio », 1994, p. 135.

${ }^{10}$ Jean Cayrol, «Témoignage et littérature », Esprit, $21^{\mathrm{e}}$ année, avril 1953, pp. 575-578.
} 
romanesque un peu gluante et poisseuse aux doigts ", contre ces romanciers, Erich Maria Remarque, Robert Merle, qui «s'attachent à donner [...] un corps romanesque à ce qui n'était qu'un monstre impossible à décrire et à comprendre, un monstre dont les flancs 'étaient animés par la douleur' suivant les termes de Dante ». Pour Cayrol, c'est une insulte que d'oser faire du camp de concentration « une image, une fiction, une fable ». Il doit demeurer dans le domaine de l'inouï, de l'indicible, c'est, dit-il, "une expérience intransmissible, solitaire, instable ». Cayrol en appelle à la pudeur, au respect. Et cette pudeur, ce respect supposent qu'on ne fasse pas «style » avec un tel sujet. Devenu matière romanesque, le camp de concentration n'est guère autre chose qu'un moyen d' « approvisionner» le public « en horreur ». Romancer le camp, c'est le « désamorcer », l'appauvrir, le ré-humaniser, en un mot, c'est trahir la mémoire de ceux qui l'ont vécu, qui y sont restés. Cayrol dénie donc à quiconque le droit de fictionnaliser l'expérience concentrationnaire. R. Bosc, commentant en 1953 dans la revue Études l'œuvre de Merle, ne disait pas autre chose :

[...] à vouloir romancer ces faits horribles, il nous semble qu'on insulte presque à la mémoire des morts, et franchement, La mort est mon métier nous paraît un livre absolument raté, sans commune mesure avec la terrible gravité du sujet qu'il prétend traiter. ${ }^{11}$

Le détour par la fiction - qui agence, sélectionne, peut se faire elliptique - pour transcrire l'histoire, cette histoire-là, est ainsi présenté comme scandaleux, insultant, face à l'effroi, à l'horreur des faits qu'il euphémise. Cet interdit est si profondément ancré qu'on le retrouve aujourd'hui sous la plume d'Emmanuel Bouju qui, analysant le roman de Merle, s'en prend aux artifices du récit, et notamment aux ellipses et sommaires qu'il refuse d'imputer au seul narrateur du récit car ils relèvent selon lui d'un choix arbitraire, celui de l'auteur, d'une « position d'écriture par laquelle dix-huit mois d'Auschwitz et son cortège effroyable de morts 'se résument' en quelques lignes $»^{12}$.

L'autre interdit repose sur le «qui peut dire?». Les écrits concentrationnaires sont des écrits pris en charge par des témoins des faits, ceux qui ont vécu la barbarie, ce sont des écrits testimoniaux. Leur légitimité, leur authenticité même proviennent de ce que le locuteur parle

\footnotetext{
${ }^{11}$ Robert Bosc, « Robert Merle, La mort est mon métier », Études, n ${ }^{\mathrm{o}} 278,86^{\mathrm{e}}$ année, Paris, juillet-août-septembre 1953, p. 137.

${ }^{12}$ Emmanuel Bouju, La transcription de l'histoire, op. cit., p. 139.
} 
d'expérience, témoigne de son vécu. Pour Lanzmann, pour Cayrol également, seul le témoin, la victime, est autorisé à parler. Celui qui n'a pas vécu les camps ne peut qu'être du côté de la fiction, donc de la transgression. Cayrol refuse à Merle le droit de prendre sur ce sujet la parole. Quelle légitimité a-t-il ? Il n'est pas un rescapé. Et on lui reprochera de n'avoir, de l'univers concentrationnaire, qu'une connaissance indirecte, livresque, historique.

Comment écrire, quelle langue utiliser pour dire la barbarie, quelle légitimité à faire entendre sa voix ? Merle s'est posé toutes ces questions, reprenant à son compte les interdits exposés précédemment, comme il le précise à Marie-Élisa Nordmann : « Je me suis demandé comment j'allais écrire ce livre, comment j'allais le faire. Et finalement j'ai compris que comme je n'avais pas été déporté, je ne pouvais pas l'écrire du point de vue des déportés $\gg{ }^{13}$.

Comme Merle le rappelle dans sa préface de 1972, son œuvre effectivement ne relève pas du témoignage direct : elle s'appuie sur des sources - le résumé des entretiens de Hœss avec le psychologue américain Gilbert et les documents du procès de Nuremberg -, sur un savoir abstrait qui s'est concrétisé par des lectures. Il n'est pas un témoin, il fait «œuvre d'historien» et s'accorde la liberté d'une «re-création étoffée et imaginative de la vie de Rudolf Hœss d'après le résumé de Gilbert » (MEMM II). Cette dimension, et fictive et historique de son œuvre, Merle la revendique, comme il revendique le droit à la parole d'un témoin indirect et le droit de « passer le témoin » aux générations à venir, comme le dirait Marie Bornand ${ }^{14}$. Faisant œuvre d'historien, Merle n'aura de cesse de gommer autant que faire se peut les effets, artifices littéraires : le style s'efface, la narration prend un aspect documentaire. Le style, dit-il, « devait être cette espèce de style neutre, impersonnel, il ne fallait surtout pas que ce soit littéraire ${ }^{15}$; Emmanuel Bouju lui reconnaît d'ailleurs cette utilisation de « procédures de neutralisation des effets du modèle narratif ${ }^{16}$.

\footnotetext{
${ }^{13}$ Claude Prévost, "Entretien avec Robert Merle et Marie-Élisa Nordmann», La déportation dans la littérature et l'art, La Nouvelle Critique, revue du marxisme militant, $\mathrm{n}^{\mathrm{O}} 167$, juin 1965, p. 52.

${ }^{14}$ Marie Bornand, Témoignage et fiction. Les récits de rescapés dans la littérature de langue française (1945-2000), Genève, Droz, 2004, p. 66.

${ }^{15}$ Claude Prévost, «Entretien avec Robert Merle et Marie-Élisa Nordmann », op. cit., p. 60.

${ }^{16}$ Emmanuel Bouju, La transcription de l'histoire, op. cit., p. 138.
} 
Face aux critiques qui reprochent à Merle ses ellipses et ses sommaires, on pourrait rétorquer avec les mots de Semprun : «[...] l'essentiel n'était pas l'horreur accumulée, dont on pourrait égrener le détail, interminablement [...]. L'essentiel [...] c'est l'expérience du Mal ${ }^{17}$. Et c'est bien cette expérience du Mal que Merle veut questionner, c'est ce qui explique sa focalisation non sur l'horreur, non sur l'effroi mais sur l'homme de devoir, monstrueux, qui inspire effroi et horreur. Et puisque Merle ne peut en rendre compte du point de vue du déporté, il fait alors un choix audacieux : il offre une voix au bourreau. Inédit.

Nous en venons à ce qui constitue sans doute l'aspect le plus scandaleux de l'œuvre, en total décalage avec la doxa de l'époque : le choix d'une narration homodiégétique, l'émergence de la parole du nazi, d'un narrateur qui est un monstre. Merle, qui offre une voix à Rudolf Hœss, qui choisit de raconter l'histoire du point de vue du bourreau, peut être considéré comme un précurseur, comme l'ont noté les critiques. Ainsi, François Genton, évoquant dans un article le film de Wolfgang Staudte, Les assassins sont parmi nous (Die Mörder sind unter uns), de 1946, le compare au roman de Merle :

Le film de Staudte en 1946 et le roman de Merle méritent d'être nommés des œuvres premières dans leur genre respectif : Les assassins sont parmi nous est la première fiction cinématographique allemande qui traite des crimes nazis, $L a$ mort est mon métier est la première tentative de donner voix aux criminels et de comprendre de leur point de vue leur cheminement et leurs actes - mais sans faire sien ce point de vue, sans en rajouter. ${ }^{18}$

Emmanuel Bouju note de même : « ce roman sera pris comme casmodèle, précédent historique sensible qui met en évidence les risques de la démarche et permet de mieux évaluer les tentatives plus récentes ${ }^{19}$.

Merle choisit donc le détour par l'autobiographie fictive de Rudolf Hœss ; un choix qui relève d'une logique de scandale, secoue les tabous de toute une époque, des tabous tellement enracinés qu'ils perdurent aujourd'hui, et révèlent un malaise qui tient sans doute plus à la posture du lecteur-interprète qu'à celle de l'auteur. La charge fut si virulente contre l'œuvre de Merle qu'en 1965 il se justifie encore de ce choix

\footnotetext{
${ }^{17}$ Jorge Semprun, op. cit.,pp. 119-120.

${ }^{18}$ François Genton, «Le Dictateur et les autres : satire première et satire seconde », Revue de littérature comparée, $\mathrm{n}^{\mathrm{0}} 324$, avril 2007, pp. 459-472. Je souligne.

${ }^{19}$ Emmanuel Bouju, La transcription de l'histoire, op. cit., p. 134. Je souligne.
} 
audacieux et de n'être pas Lang. L'écriture, précise-t-il, a été douloureuse tant ces deux voix, celle de l'auteur, celle du narrateur, sont antagonistes :

[...] je suis évidemment l'anti-Lang, je suis tout le contraire de Lang à tous points de vue. [...] c'était très difficile de me couler dans l'esprit d'un homme de ce type. [...] Bien entendu, je n'ai pas vécu tout cela comme un problème de style, mais comme un problème moral. J'ai dû me soumettre à la discipline de porter ce Lang en moi. Et cela a été horriblement dur. [...] Je me suis brimé incroyablement en faisant cela. ${ }^{20}$

Cette première personne confiée à un bourreau, qui a fait et fait encore couler beaucoup d'encre, est un véritable tour de force: se restreindre au point de vue du SS, c'est, pour Merle, orchestrer son effacement et se refuser à adopter une perspective surplombante. C'est s'amputer :

J'ai voulu écrire le livre à la première personne pour qu'à aucun moment ce qu'il y a en moi de chaleureux, de coloré, n'arrive à transparaître. Il fallait que ma personnalité ne s'interpose pas comme un écran entre le personnage qui dit je et le lecteur. Que ce personnage qui dit je ne soit jamais moi, n'ait jamais mes réactions, soit toujours Rudolf Lang. ${ }^{21}$

S'il délègue la narration à son personnage, c'est que «ce 'je' [est] en réalité un 'il' pour lequel [il] éprouv[e] une profonde horreur $»^{22}$. Et cette ascèse qu'il s'impose est à dessein perturbante: pas de «il» distancié et confortable, ni pour lui, ni pour le lecteur, mais un « je » qui impose le rejet, un je-rebut, cet autre qu'il se refuse à être.

Pour certains critiques contemporains de Merle, c'est justement cette narration à la première personne, cette voix du bourreau, enfin audible, qui fait la qualité de l'œuvre. C'est la position d'Aragon, par exemple, qui considère que «le grand mérite de ce livre est là-même $»^{23}$. Aragon, qui fit l'éloge de «l'auteur de La mort est mon métier qui, en donnant ce portrait saisissant du tueur nazi, a su démontrer l'usage moral qui peut valablement être fait de la connaissance du mal $»^{24}$, considère que ce roman aide à voir le « haïssable en le connaissant». Quant à la

\footnotetext{
${ }^{20}$ Claude Prévost, «Entretien avec Robert Merle et Marie-Élisa Nordmann », op. cit., p. 60 .

${ }^{21}$ Robert Merle, lettre à Madame Köves, Budapest, archives privées, p. 4.

${ }^{22}$ Robert Merle, «Le jeu des je dans L'Idole », archives privées.

${ }^{23}$ Louis Aragon, La lumière de Stendhal, Paris, éditions Denoël, 1954, p. 147 puis 154.

${ }^{24}$ Ibid., p. 120.
} 
question de l'absence de contrepoint positif dans l'œuvre, Aragon précise : «Le livre refermé, je n'ai pour ma part aucunement éprouvé comme un manque l'absence, face à ce monstre, du héros positif ${ }^{25}$. Dans La Pensée, en 1953, Marc Soriano consacra lui aussi un long article à l'œuvre de Merle et, s'il nota que «la technique littéraire de 'la première personne', visiblement choisie avec soin par l'auteur, présente l'inconvénient de réduire à des touches rapides le portrait des personnages positifs » ${ }^{26}$, il ne tarit pas d'éloge sur «l'œuvre de maturité d'un grand artiste », une œuvre « capable d'aider à la prise de conscience politique du lecteur et par ricochet de rendre plus complète celle de son auteur $»$.

Marie-Élisa Nordmann, rescapée d'Auschwitz, indique avoir été particulièrement frappée lors de la parution de l'œuvre par «cette explication de la psychologie des S.S qu'[elle] cherchai[t] depuis [s]on retour $»{ }^{27}$; « la psychologie de l'homme qui était arrivé à faire cela, a été pour [elle] une révélation ${ }^{28}$. Elle étend cet impact à l'ensemble des déportés : «Je peux dire que ce livre a beaucoup intéressé les déportés parce qu'il exprimait la mise en place de ce dont ils avaient été témoins : l'extermination massive, en expliquant la psychologie de celui qui l'avait organisée $\gg{ }^{29}$.

Mais Merle, on le sait, fut éreinté par la critique, attaqué de biais pourrait-on dire puisque selon ses propres termes: «On ne m'a pas attaqué sur le contenu. Ça c'est la règle. C'est le contenu qui choque, mais c'est la forme qu'on attaque. Toujours $»{ }^{30}$. Nadeau lui reprocha le manque d'ampleur de ce qui n'était même pas une fresque; sous l'impulsion de Nimier, on critiqua la multiplication d'expressions allemandes dans le texte, procédé jugé artificiel, que Merle utilisa pour, dit-il «[introduire] le lecteur [...] dans l'univers mental des Allemands $[\ldots] » »^{31}$.

L'œuvre de Merle fut donc bien une œuvre à contre-courant, qui trouva peu de lecteurs en 1952, ne fut publiée en version de poche qu'en

\footnotetext{
${ }^{25}$ Ibid., p. 156.

${ }^{26}$ Marc Soriano, « Le typique et ses techniques à travers quelques romans récents », La Pensée, no 48-49, mai-juin-juillet-août 1953, pp. 223-224.

${ }^{27}$ Claude Prévost, «Entretien avec Robert Merle et Marie-Élisa Nordmann », op. cit., p. 56.

${ }^{28}$ Ibid., p. 64.

${ }^{29}$ Ibid., p. 64

${ }^{30}$ Ibid., p. 56.

${ }^{31}$ Sur ces critiques, évoquées par Merle, voir l'entretien avec Marie-Élisa Nordmann.
} 
1961, soit neuf ans après sa parution, et ne connut véritablement le succès que lorsqu'elle fut considérée comme un livre d'histoire. On comprend mieux pourquoi l'auteur ressentit la nécessité en 1972 d'écrire une préface pour justifier ses choix, historiciser son roman et fournir en quelque sorte des consignes de lecture. Il peut sembler étonnant, vingt ans après, de l'entendre dire de cette œuvre que «c'est un livre d'histoire » (MEMM I), de ne la voir jamais qualifiée de « roman » - il opte pour le substantif "livre» - alors que lors de son entretien avec Marie-Élisa Nordmann, le terme « roman » lui échappe parfois. Mais, de fait, le statut du livre a changé. La forme romanesque, encore revendiquée par Merle en 1965 et choisie pour son "pouvoir de rayonnement», pour sa «puissance de choc» bien supérieurs à ceux d'un essai historique ${ }^{32}$, si elle a permis que perdure l'œuvre, s'est finalement estompée au profit de son impact historique. Or, il nous semble que la fiction n'enlève rien à la connaissance des faits, qu'elle peut même rendre lisibles les incontournables documents et témoignages. Déjà, en 1965, Marie-Élisa Nordmann indiquait que le témoignage direct, vingt ans après les faits, s'il était toujours indispensable, devait être relayé par la fiction :

$\mathrm{Au}$ début, nous, les rescapés, nous avons écrit des témoignages, fait des conférences, etc., sur les événements que nous avions vécus [...]. Mais cela ne suffit pas. On ne peut plus se satisfaire de témoignages purs et cela ne satisfait pas non plus ceux que l'on essaie de toucher.

Selon elle, pour «faire sentir maintenant ce qu'était la déportation », et ce face à des jeunes gens pour lesquels la déportation relève de l'histoire, «La mort est mon métier nous aide considérablement $»^{33}$.

\section{L'effet Littell sur le Merle précurseur}

Merle est revenu sur le devant de la scène lors de la polémique de 2006 autour de la parution des Bienveillantes de Jonathan Littell. Le succès est phénoménal, le roman est couronné par le Goncourt et le grand prix du roman de l'Académie Française ; on avance même qu'il inaugure l'ère des bourreaux. L'œuvre enflamma la critique, et notamment la

\footnotetext{
${ }^{32}$ Ibid., pp. 55-56.

${ }^{33}$ Ibid., p. 56.
} 
critique universitaire : la controverse fut virulente, opposant de manière souvent passionnelle, les pro et les anti Littell. Devant la première personne dérangeante de Max Aue, le SS de Littell, devant ce roman du bourreau nazi, devant la rupture avec la tradition de l'expérience concentrationnaire relatée par des écrivains témoins, on s'est remémoré le Rudolf Lang de Merle. C'est pourquoi nous nous arrêterons un instant sur cette polémique, sans viser l'exhaustivité, car là n'est pas notre propos.

La polémique s'enflamme dès l'automne $2006^{34}$ et, plus d'un demisiècle après La mort est mon métier, les mêmes arguments vont être avancés pour critiquer l'œuvre, des arguments d'ordre littéraire, d'ordre moral et historique. Lanzmann, tout d'abord, dans Le Journal du dimanche, s'insurge face au «paradoxe inimaginable des Bienveillantes » qui réside selon lui dans le fait que «la tâche de mémoire, d'intellection et de récit de la Shoah est confiée à un SS, qui parle pendant plus de neuf cents pages ${ }^{35}$; il dénonce la séduction du bourreau que ce type de narration suscite. Les historiens, Peter Schöttler, Florent Brayard, Edouard Husson, pistent les invraisemblances et mettent en cause dans le roman les défaillances d'ordre historique. Les littéraires dénoncent à la manière de Cosmin Toma, «l'artifice littéraire le plus éhonté. En effet, la rhétorique du 'je' obnubilant ses lecteurs naïfs, telle qu'elle est mise en scène dans le roman, nous plonge immédiatement dans les eaux troubles de la manipulation et du Mal, sous leur forme la plus littéraire $[\ldots] \gg{ }^{36}$; Charlotte Lacoste, intarissable sur le sujet dans les années qui suivent, condamne de manière catégorique Les Bienveillantes qui offre pour elle un «procès en réhabilitation du bourreau » dont on cherche à « redorer le blason », qui ouvre une « ère

\footnotetext{
${ }^{34}$ Voir à ce sujet le résumé de la réception critique du roman par Richard J. Golsan, «Les Bienveillantes et sa réception critique: littérature, morale, histoire », Marc Dambre, Richard J. Golsan (éd.), L'exception et la France contemporaine. Histoire, imaginaire, littérature, Paris, Presses Sorbonne nouvelle, 2010, pp. 45-56; voir également la mise au point de Jean Solchany, "Les Bienveillantes ou l'histoire à 1'épreuve de la fiction ", Revue d'histoire moderne et contemporaine, $\mathrm{n}^{\circ}$ 54-3, mars 2007, pp. 159-178, [En ligne].

${ }^{35}$ Claude Lanzmann, «Les Bienveillantes, vénéneuse fleur du Mal», Le Journal du Dimanche, $\mathrm{n}^{\circ} 3114,17$ septembre 2006.

${ }^{36}$ Cosmin Toma, «Les Bienveillantes et les limites de la littérature », Ugo Dionne et Michel Fournier (dir.), Les lieux de la réflexion romanesque au XVIII siècle: de la poétique du genre à la culture du roman, Études françaises, vol. 49, numéro 1, 2013, pp. 167-180, [En ligne].
} 
du nazisme décomplexée $»^{37}$ et va, dans l'abjection, au-delà même du révisionnisme : «c'est pire que de nier, il s'agit de rendre agréable ${ }^{38}$. Cet argument, elle le reprend à François Rastier qui, dans un article au titre accrocheur : "Croc de boucher et rose mystique - Enjeux présents $\mathrm{du}$ pathos sur l'extermination », dénonce de manière virulente la littérature du « saignant » :

\begin{abstract}
Affichant leur prétention historique voire documentaire, s'appuyant sur des entretiens avec des cadres nazis (Merle), des témoignages de victimes (J-F Steiner), des dossiers historiques (J-F Steiner, Littell), ces romans ont en commun de donner la parole aux bourreaux, de laisser cours à leur monologue intérieur et de déployer un érotisme teinté de sado-masochisme. Ils transforment l'extermination en matière fabuleuse (comme jadis la Matière de Bretagne), relevée de pathos pour appâter le public. [...] ces nouveaux poncifs du mélo s'appuient sur les deux grands ressorts populaires du pathos, le palpitant et le saignant. ${ }^{39}$
\end{abstract}

Rapidement et pour reprendre les différents arguments, qui dépassent largement ceux qui furent avancés en 1952, vue la spécificité de l'œuvre de Littell, on dénonce chez ce dernier une fascination pour l'horreur, un penchant certain pour le voyeurisme, des invraisemblances et notamment cette mise en scène d'un héros SS cultivé, un esprit (trop) subtil, qui a la double nationalité française et allemande; son histoire familiale, agrémentée d'inceste, d'une gémellité névrotique, son homosexualité, sa criminalité - il tue mère, beau-père, ami manqueraient de crédibilité et lui confèreraient un statut hors norme qui n'aurait que peu à voir avec le portrait type du SS. Sa logorrhée, l'épanchement sur neuf cents pages, serait en totale contradiction avec le silence refuge des SS. On dénonce aussi le pacte pervers que le

\footnotetext{
${ }^{37}$ Sur les outrances de Charlotte Lacoste, on se reportera à l'article de Luc Rasson, «De la critique littéraire considérée comme un exercice de mépris ", Acta fabula, vol. 14, $\mathrm{n}^{\mathrm{o}} 5$, « L'aire du témoin », juin-juillet 2013, [En ligne].

${ }^{38}$ Charlotte Lacoste, «L'extermination comme matière fabuleuse : Les Bienveillantes ou l'art de rendre le nazi fréquentable », Paroles gelées, 24 (1), 2008, respectivement pp. 19, 25, 14, [En ligne] ; voir également du même auteur : « Un cas de manipulation narrative : Les Bienveillantes », vol. XIV - $\mathrm{n}^{\circ}$ 1, Coordonné par Évelyne Bourion, 2009, [En ligne]; «Le roman sied-il à l'extermination », Villa Europa, $\mathrm{n}^{\circ} 2$, Université de Saarbrucke, 2011, [En ligne]; « De la vigilance critique », Philippe Mesnard et Yannis Thanassekos, Témoigner. Entre histoire et mémoire, 100, juillet-septembre 2008, pp. 61-65, [En ligne] ; Séductions du bourreau, Paris, Presses Universitaires de France, coll. « Intervention philosophiques », 2010.

${ }^{39}$ François Rastier, « Croc de boucher et Rose mystique - Enjeux présents du pathos sur l'extermination », Texto! Textes et cultures, XII, 2, avril 2007, pp. 1-27, [En ligne].
} 
romancier impose au lecteur et qui rendrait ce dernier complice, pacte qui nous invite à fraterniser avec le bourreau.

Le roman, nous l'avons dit, fut aussi défendu, parfois de manière hyperbolique, parfois avec retenue et réserve : on salua, à la manière de Julia Kristeva, la « connaissance - sans précédent littéraire - des travaux sur le déroulement des opérations de guerre, aussi bien que sur l'organisation et la pratique de la 'solution finale', et, davantage encore », et « la virtuosité d'une narration qui insère l'histoire dans la psychopathologie d'un homme ${ }^{40}$. Semprun, membre du jury Goncourt, s'est déclaré satisfait de l'obtention de ce prix par Littell et Élie Wiesel, qui n'a pas lu Les Bienveillantes, s'est cependant exprimé sur la liberté des romanciers qui, selon lui, «peuvent tout se permettre » ${ }^{41}$, il en va ensuite de leur responsabilité s'ils travestissent la réalité. Pour lui, témoignage brut et fiction ne sont pas antagonistes, loin de là, ils peuvent être complémentaires :

Jamais on ne dépassera la force d'un document, de journaux, de témoignages écrits. Jamais, on ne pourrait atteindre la profondeur du désespoir de celui qui l'a vécu [...]. Mais le roman peut aider la mémoire, il peut entretenir la flamme. On peut aussi construire des fictions à partir de ces documents. Libre à l'écrivain de le faire.

Il ne condamne pas la littérature des bourreaux et comprend ce qui la suscite : « Mais, à travers la fiction, on peut aussi se poser la question 'quel genre d'homme était le tueur ?' On peut 'entrer' chez lui [...]. On se rend compte ainsi que le bourreau n'est pas quelqu'un qui vient de l'au-delà. Mais d'ici ».

Cette polémique sur laquelle nous nous sommes attardée a atteint rétrospectivement Robert Merle. Le plus souvent, il fut juste cité, au passage. Il fut parfois associé au discrédit global que suscite la littérature $\mathrm{du}$ bourreau, comme nous l'avons vu avec François Rastier. La psychanalyste Régine Waintrater considère que la visée des deux auteurs est la même : "prétendre pénétrer à l'intérieur du mal, en nous révélant

\footnotetext{
${ }^{40}$ Julia Kristeva, «De l'abjection à la banalité du mal», Conférence avec Jonathan Littell auteur du roman Les Bienveillantes, invité par le Centre Roland Barthes (Université Paris-VII), à l'ENS, le mardi 24 avril 2007, avec Julia Kristeva et Rony Brauman, [En ligne].

${ }^{41}$ Pour cette citation et les suivantes : Élie Wiesel, « Le roman peut aider la mémoire », interview par Mohammed Aissaoui, le Figaro.fr, 10 novembre 2010, [En ligne].
} 
les ressorts intimes du bourreau, ainsi promu à la dignité d'interlocuteur privilégié $\gg{ }^{42}$.

Mais il arrive que Merle soit distingué de Littell. C'est le cas par exemple dans l'article d'Éric Levéel, qui évoque cette divergence tout d'abord en opposant la sexualité perverse de Max Aue et le bon père de famille, l'homme ordinaire, qu'est Rudolf Lang, ensuite en montrant que les démarches des deux auteurs sont divergentes, transcription d'un côté, fiction de l'autre :

[...] si les deux auteurs ont pour personnages principaux les bourreaux plutôt que les victimes vues comme des masses plus ou moins informes [...], la comparaison semble s'arrêter là tant Rudolf Lang et Maximilien Aue sont antinomiques, tant la démarche de Littell est divergente de celle plus historique, plus documentaire de Robert Merle qui se contente de transcrire plus ou moins fidèlement les comptes rendus du procès de Rudolf Höß à Nuremberg et les entrevues avec le psychiatre commis d'office, le tout de manière romancée. Chez Littell, outre la différence fondamentale entre Aue et Lang, il s'agit d'une véritable œuvre de fiction $[\ldots]^{43}$

Philippe Lançon dans Libération avait, dès le 10 novembre 2006, mis en valeur, par contraste, la retenue de Merle :

Son roman est terrible et, pour cette raison, animé d'une grande pudeur. Jamais l'auteur ne songerait à mêler son ego à ce qu'il décrit. L'idée d'en rajouter lui paraîtrait obscène. Les temps ont changé. La foule a faim. Pour 25 euros, le buffet aux horreurs lui est ouvert. Comme au Club Méditerranée, il est illimité. Désormais, comprendre c'est manger.

\section{Voix de salaud : lire contre ${ }^{44}$}

Merle, nous l'avons vu, avant Littell, bouleverse donc une tradition, s'attaque à un tabou : la littérature concentrationnaire est une littérature qui donne voix aux victimes, qui se fait du point de vue des victimes. Et quand le bourreau, et qui plus est un bourreau historique, dit « je », se pose bien sûr le problème de l'identification, de l'empathie, le

\footnotetext{
${ }^{42}$ Régine Waintrater, «Les Bienveillantes, intimité forcée ou intimidation », Témoigner. Entre histoire et mémoire, op. cit., pp. 53-60, [En ligne].

${ }^{43}$ Éric Levéel, « Maximilien Aue : une homosexualité de rigueur? », Les Bienveillantes de Jonathan Littell, Études réunies par Murielle Lucie Clément, Londres, [En ligne], Openbook Publishers, 2010, pp. 141-153.

${ }^{44}$ Nous empruntons l'expression à Luc Rasson, "Lire contre», Paroles de salaud: Max Aue et Cie, Études réunies par Luc Rasson, CRIN, n ${ }^{\circ}$ 58, Amsterdam, Rodopi, 2013, pp. 7-15.
} 
problème du « qui parle? » et du lien entre le « je » d'un Hœss et celui d'un Lang de fiction.

L'œuvre de Merle est orchestrée autour d'une véritable bi-partition, comme il le précise dans sa préface: une "re-création étoffée et imaginative de la vie de Rudolf Hœss » (MEMM II) dans une première partie ; une « œuvre d'historien » dans une seconde.

Dans son entretien avec Marie-Élisa Nordmann, Merle insiste sur sa documentation scrupuleuse, sur le long travail qu'il a effectué en amont de la rédaction de l'œuvre : documentation sur les Corps Francs, sur la Turquie en 1916, accès aux archives de Nuremberg au Centre de Documentation Juive Internationale ${ }^{45}$, reconstitution à partir de ces archives de la manière dont Hœss a conçu l'usine de mort. Au Centre de documentation, il dit avoir été "stupéfait d'être le seul chercheur à regarder, à consulter ces photos, ces documents impressionnants, ces photocopies, ces rapports hallucinants, incroyables des SS sur les exécutions massives » ${ }^{46}$. Merle eut accès également aux «Témoignages sur Auschwitz», publiés par l'amicale des déportés d'Auschwitz fin 1946. Et de fait, l'œuvre, qui nous permet de suivre le parcours de HœssLang de 1913 à 1947, nous propose, comme le note Marc Soriano, une évocation historique ample et riche, " évocation historique non du peuple allemand, mais, à travers un monstre caractéristique, des conditions économiques, politiques, sociales et psychologiques qui rendirent possible le succès du nazisme $\gg{ }^{47}$. Précurseur en la matière, Merle propose donc la toute première exploitation romanesque d'une analyse des ressorts, des mécanismes idéologiques, psychologiques, sociauxéconomiques du nazisme ; la première reconstitution romanesque de la genèse de l'usine de mort également.

Dans le même temps, il se permet une «re-création » de la vie de Hœss. Quelles sont alors ses sources? Il en a peu. La confession de Hœss, rédigée dans la prison de Cracovie avant son procès, ne sera publiée qu'en $1959^{48}$, soit sept ans après la fiction de Merle. Pour le

\footnotetext{
${ }^{45}$ Centre de Documentation Juive Contemporaine, CDJC, installé, à partir de 1951, rue Guénégaud à Paris, comme l'indique Merle.

${ }^{46}$ Claude Prévost, «Entretien avec Robert Merle et Marie-Élisa Nordmann », op. cit., p. 54.

${ }^{47}$ Marc Soriano, «Le typique et ses techniques à travers quelques romans récents », article cité, p. 223.

${ }^{48}$ Rudolph Hœss, Le commandant d'Auschwitz parle, première publication chez Julliard, 1959. Notre édition de référence sera celle de La Découverte/Poche, 1995, 2005 pour la postface.
} 
reste, Merle s'est appuyé sur les dépositions de Hœss lors du procès de Nuremberg et sur les écrits du psychologue américain Gilbert qui servit d'interprète lors du procès et qui interrogea longuement le bourreau d'Auschwitz dans sa cellule et obtint de ce dernier une "première version qui était sincère et vraie de sa vie ${ }^{49}$. Merle explique que l'intégralité de la confession ne fut pas publiée par Gilbert et que ce dernier lui a envoyé « dix pages dactylographiées à double interligne » ${ }^{50}$ à partir desquelles il a écrit son roman. C'est pourquoi l'auteur de $L a$ mort est mon métier indique : "paradoxalement, l'autobiographie écrite par Hœss lui-même est infiniment moins véridique que celle que j'ai écrite ${ }^{51}$. Aux mensonges de Hœss qui écrivit pour se justifier sur le conseil de ses avocats, qui occulta bien des aspects de sa trajectoire, « pour cacher les choses qui le gênaient ${ }^{52}$, Merle oppose donc sa recréation étoffée à partir des documents de Gilbert, qui aurait atteint, par la psychologie, la vérité de Hœss.

On reprocha beaucoup à Merle cette psychologie lors de la parution du roman et en pleine polémique Littell. Régine Waintrater dénonce chez Merle, le "piège d'un psychologisme réducteur qui conforte le lecteur dans l'idée qu'il a pénétré la genèse du mal absolu $»^{53}$. Emmanuel Bouju avance que c'est «le privilège du romancier démiurge, de s'arroger l'identité de son Amphitryon historique pour s'établir au cœur de son espace mental et se l'approprier » pendant que « le vrai Amphitryon reste absent de la scène. Il n'existe plus que dans la mémoire historique $[\ldots] »^{54}$. Merle tendrait donc à générer une confusion entre le vrai et le faux Amphitryon dont il s'arroge l'identité. Lang, personnage de fiction - auquel Merle a décidé de conférer une identité fictive, avec ce patronyme courant, banal, sans connotation $^{55}$ - est-il ou n'est-il pas

\footnotetext{
${ }^{49}$ Claude Prévost, « Entretien avec Robert Merle et Marie-Élisa Nordmann », op. cit., p. 53.

${ }^{50}$ Ibid. Pour les documents de Gilbert, voir Gustav Mark Gilbert, « Psychologie de la dictature : Frank, Keitel, Hoess », Les Temps Modernes, $10^{\mathrm{e}}$ année, $\mathrm{n}^{\mathrm{0}} 107$, novembre 1954, pp. 696-712, pour « Le colonel SS Hoess d'Auschwitz ».

${ }^{51}$ Ibid.

${ }^{52}$ Ibid.

${ }^{53}$ Régine Waintrater, «Les Bienveillantes, intimité forcée ou intimidation », article cité, p. 57.

${ }^{54}$ Emmanuel Bouju, La transcription de l'histoire, op. cit., pp. 145-146.

${ }^{55}$ La patronyme «Lang » n'est toutefois pas totalement fictif, il correspond à l'identité usurpée de R. Hœss, en fuite dans l'île de Sylt sous le nom du quartier-maître Franz Lang, avant d'être arrêté en mars 1946 alors qu'il se fait passer pour agriculteur.
} 
Hœss, Amphitryon historique? Est-il une falsification du Hœss historique?

Merle revendique explicitement l'attache historique : son Lang est Hœss. Mais l'Amphitryon de fiction, ce Lang dont il retrace, recrée le parcours, dont il pénètre la conscience, peut séduire, selon Bouju, et Merle court alors le risque «que les charmes du faux Amphitryon ne fassent oublier le vrai » ${ }^{56}$. Cette séduction tiendrait aux «clés d'interprétation psychologique $»^{57}$. Ainsi donc le reproche fait à l'auteur serait celui de prétendre faire œuvre d'historien « en usant d'autres moyens que ceux du discours historiographique $"{ }^{58}$ au risque d'un glissement de l'histoire à la fiction et de la fiction à la manipulation. Et Bouju, et Waintrater également, de critiquer les pages consacrées dans l'œuvre à l'enfance traumatique, au père tyran, le choix du récit de vie et la présentation de l'Histoire, cette Histoire-là, en lien étroit avec l'histoire individuelle du bourreau. Si l'on considère que la première partie du roman, la «re-création », s'étend sur cinq chapitres, de « 1913 » à « 1934 » - la date de 1934 marquant la décision d'Himmler de nommer Lang à un poste dans l'administration du Konzentrationslager de Dachau (MEMM 227) - soit plus de deux cents pages, il est clair que Merle a largement étoffé les dix pages de Gilbert. Il va de soi que, si l'on n'a pas accès aux documents de Gilbert, si l'on prend comme référence douteuse - l'autobiographie de Hœss, alors certes, les quatre premiers chapitres de Merle peuvent apparaître comme une falsification de l'Amphitryon historique puisque bon nombre d'éléments insérés par Merle ne figurent pas dans le texte de Hœss et semblent relever de l'affabulation. Ainsi, si Hœss insiste effectivement sur l'attachement fanatique de son père à la religion, il ne fait nullement mention d'une quelconque maladie de son père, vécue comme un véritable châtiment pour avoir fauté et qui développa chez lui un sentiment de culpabilité tel qu'il passa sa vie ensuite à tenter de racheter sa faute. D'où, selon Merle le fanatisme religieux, d'où le choix imposé à son fils d'entrer en religion. Merle a eu connaissance de ces faits au travers des analyses de Gilbert et a choisi de les intégrer à son roman ${ }^{59}$. De même, pour défendre

\footnotetext{
${ }^{56}$ Emmanuel Bouju, La transcription de l'histoire, op. cit., p. 145.

${ }^{57}$ Ibid., p. 139.

${ }^{58}$ Ibid., p. 146.

${ }^{59}$ Ainsi peut-on lire dans le texte de Gilbert, suite à une longue citation de Hœss expliquant la bigoterie de son père, sa "piété fanatique » l'analyse suivante : "C'est ainsi que le jeune Hœss se vit imposer le rôle de l'être consacré à Dieu par un père fanatique qui expiait quelque remords - probablement pour avoir contacté la syphilis (la
} 
ce qui a pu passer pour invraisemblable dans son œuvre (le fait qu'Elsie, la femme de Lang, ne découvre ses activités véritables que tardivement), Merle s'appuie sur Gilbert, ou plutôt sur les dires de Hœss à Gilbert : « c'est à cette date [1943], à la suite d'un mot qui a échappé à un visiteur, que ma femme a compris $»{ }^{60}$. Ainsi, dans l'œuvre, le souci historique, qui apparaît notamment dans la reprise de phrases prononcées par Hœss luimême lors du procès de Nuremberg, se conjugue au souci de vérité qui, pour Merle, passe par le détour de la psychologie ; le « liant» est ensuite assuré par la fiction pure. L'auteur a donc recréé - à partir d'un matériau qu'il juge véridique, les écrits de Gilbert - un Hœss qu'il juge plus vrai que celui-là qui se peint largement de profil dans sa propre confession.

C'est à ce Hœss-Lang-là que Merle choisit de donner une voix. Et Emmanuel Bouju s'interroge : "pourquoi donner voix, pourquoi aller jusqu'à donner $s a$ voix au bourreau ? ${ }^{61}$ S'il est possible d'adhérer à la première interrogative qui, moralement et littérairement, est lourde d'enjeux, nous rejetons la seconde qui part du postulat que Merle donne $s a$ voix au bourreau. L'expression nous paraît tendancieuse malgré ou à cause de l'italique qui, si elle distancie, marque aussi l'adjectif possessif d'une intensité nouvelle, le détache du corps du texte, insiste donc sur l'identi(ci)té auteur/bourreau; donner sa voix, c'est manifester un accord, c'est suggérer une adhésion entre celui qui écrit et celui qui parle, or le je narrant n'est en rien le je écrivant, comme d'ailleurs le concède Emmanuel Bouju lors de son analyse de l'épigraphe.

Il me semble, si nous voulons poursuivre sur la même problématique vocale, qu'il serait plus judicieux dans le cas de Merle de parler de voix in et de voix off. Car, de fait, il y a dans l'œuvre coexistence de deux voix antagonistes, une véritable polyphonie : la voix in est celle du narrateur, elle est dans le texte, elle est le texte même à la première personne, c'est la voix du bourreau; la voix off s'ancre elle aussi dans le texte, à rebours de la voix in, elle est intégrée, en

nature de la maladie est inconnue). Le milieu de son enfance provoqua progressivement en lui de la répulsion à l'égard de la religion et, d'une façon générale, une personnalité plutôt renfermée et docile ». Gustav Mark Gilbert, « Psychologie de la dictature : Frank, Keitel, Hœss », article cité.

${ }^{60}$ Cité par Robert Merle dans «Entretien avec Robert Merle et Marie-Élisa Nordmann », article cité, p. 58. Ce point est d'ailleurs confirmé par Léon Goldensohn, Les entretiens de Nuremberg, traduit de l'anglais par Pierre-Emmanuel Dauzat, Paris, Flammarion, coll. " Champs histoire », 2009.

${ }^{61}$ Emmanuel Bouju, La transcription de l'histoire, op. cit., p. 134. 
surimpression, c'est une voix autre, celle de l'auteur ${ }^{62}$. Ruth Amossy parlerait ici d'ethos auctorial et de son rapport problématique avec l'ethos du narrateur ${ }^{63}$. Dans le roman de Merle, comme dans celui de Littell, que Ruth Amossy analyse dans un article, l'ethos auctorial parait insaisissable tant le texte lui-même, à la première personne, est - et n'est que - pris en charge par le narrateur, par un point de vue unique, ce qui relève bien du pacte même de l'autobiographie fictive. C'est, systématiquement et à tout moment du récit, Rudolf Lang qui observe, présente les événements de manière distanciée, multipliant les détails dans un souci maniaque et ce, sans manifester le moindre sentiment. C'est d'ailleurs pour cela même que Merle opte pour une première personne, afin de ne pouvoir «manifester [son] indignation, ni [son] horreur, ni [sa] haine de ce qu'il avait fait», afin que « le regard du 'je' soit un regard qui décolore l'univers » et, pour ce faire, il fallait «que tout parût plat, que tout fût morne et pédestre, que tout soit prosaïque, il fallait vraiment un hiver conventionnel $»^{64}$.

Mais pourtant, la figure auctoriale, la voix off de Merle, s'insinue. L'antiphrase et l'ironie que Merle manie selon Soriano «avec un art souverain ${ }^{65}$, empêchent toute fusion des voix, des éthos antagonistes. Soriano l'explique parfaitement avec sa formule qui résume ce qu'il appelle l'art de Merle : «il peint l'envers pour suggérer l'endroit, le négatif pour imposer le positif $»{ }^{66}$, et la confrontation de ces deux pôles antagonistes, de ces deux voix, fait que la perspective politique est claire. Cette clarté apparaît d'ailleurs dès l'épigraphe de l'auteur qui sépare radicalement le je-auteur du je-narrateur qui s'affiche dans le titre même de l'œuvre « La mort est mon métier ${ }^{67}$. Sans la moindre ambiguïté, en

\footnotetext{
${ }^{62}$ À ce propos, Merle indiquait : « Il y a toujours derrière le texte l'auteur en filigrane avec sa protestation muette [...]», Pierre Daix, «L'Histoire est-elle un sujet de roman? », Entretien Pierre Daix - Robert Merle, Les Lettres Françaises, n ${ }^{\circ}$ 934, 11 juillet 1962 .

${ }^{63}$ Sur cette question du rapport entre éthos auctorial et éthos du narrateur, voir l'article de Ruth Amossy qui s'appuie sur Les Bienveillantes de Littell : Ruth Amossy, «La double nature de l'image d'auteur ", Michèle Bokobza Kahan, Amossy Ruth (dir.), Ethos discursif et image d'auteur, Argumentation et Analyse du Discours, [En ligne], 3 | 2009.

${ }_{64}$ «Entretien avec Robert Merle et Marie-Élisa Nordmann », article cité, p. 55 puis 60.

${ }^{65}$ Marc Soriano, «Le typique et ses techniques à travers quelques romans récents», article cité, p. 124.

${ }^{66}$ Ibid.

${ }^{67}$ Sur l'épigraphe, voir l'analyse de Emmanuel Bouju, La transcription de l'histoire, op. cit., pp. 134-135.
} 
son nom propre, Merle dédie son livre «aux victimes » et rejette les bourreaux, « ceux pour qui la Mort est un Métier».

La voix du bourreau est donc à lire comme le repoussoir ; il s'agit, comme le dit Luc Rasson, de "lire contre ». Le statut du narrateur, SS, bourreau, monstre, suffit à discréditer sa parole et dès lors « une lecture identificatrice avec le protagoniste du récit est irrecevable ${ }^{68}$. Ces salauds «que le pouvoir de la littérature dote d'une voix dérangeante et puissante à l'occasion [...] exigent du lecteur la plus élémentaire des compétences interprétatives, celle de résister aux charmes séducteurs du langage, celle de dire non ${ }^{69}$. La question finalement, la seule question sans doute à se poser devant ces romans dont le narrateur est un salaud, est, comme le dit Luc Rasson, «comment les lire ?». Car ces textes imposent, précise-t-il, « une hygiène de lecture » et nécessitent, derrière le in, d'entendre le off. S'il est un procès à faire, c'est donc sans doute plus celui du lecteur-interprète que celui de l'auteur dont l'intention ne peut être mise en question.

Car la voix off de Merle se détecte au travers du portrait du narrateur, Rudolf Lang. Tout est fait ici pour que le personnage psychorigide ne soit en rien séduisant, au sens premier, pour qu'il rebute. Ce portrait, cette voix glaciale, celle du SS criminel, monstrueux, et sans la moindre empathie, suffisent à discréditer le narrateur et à empêcher toute séduction. Pour Merle, faire entendre cette voix-là, c'était tabler sur les réactions du lecteur face à un narrateur dont tout - les valeurs, le comportement - est aux antipodes de son propre répertoire émotionnel. Ainsi, s'il y a empathie - on appréhende le personnage de l'intérieur - il n'y a pas de confusion possible entre soi et lui ; ce qui se met en place est une relation cognitive et non pas une relation affective.

Les émotions de Lang ne se propagent pas au lecteur, pour la simple et bonne raison qu'il en est dénué et que, selon nous, loin d'être confiné dans un état de sidération, comme l'évoque Ruth Amossy ${ }^{70}$, c'est-à-dire un choc émotionnel qui suspend toute faculté de juger, le lecteur, passé le temps de la stupeur, se révolte et rejette.

La voix du bourreau n'est pas une voix en contrepoids, c'est une voix en contrepoint, un contrepoison: elle vise à combattre, pas à équilibrer, elle est la voix glaciale de l'inouï... Mais sur ce point, nous

\footnotetext{
${ }^{68}$ Ibid., p. 10.

${ }^{69}$ Ibid., p. 13.

${ }^{70}$ Ruth Amossy, « La double nature de l'image d'auteur », article cité.
} 
accorderons qu'il est des lecteurs qui ne possèdent pas ce que Luc Rasson appelle « une hygiène de lecture », c'est bien pourquoi la lecture, le commentaire de telles œuvres sont indispensables, c'est bien pourquoi il faut apprendre à lire à rebours ces romans qui peignent l'envers pour suggérer l'endroit, il faut commenter, faire découvrir ces auteurs qui, comme le disait Aragon à propos de Merle, ont «pris pour règle de savoir le mal non pour mal faire, - mais pour connaître le contraire ${ }^{71}$. $\mathrm{C}^{\prime}$ est donc à juste titre que Merle, répondant à Chancel $^{72}$ qui lui demandait en 1989 lequel de ses romans il retiendrait, opta pour La mort est mon métier, un choix pédagogique, celui d'un livre dont il disait qu'il était important qu'il soit lu, un livre dont il rêvait qu'il soit étudié dans toutes les écoles.

\footnotetext{
${ }^{71}$ Louis Aragon, La lumière de Stendhal, op. cit., p. 132.

${ }^{72}$ Radioscopie, "Robert Merle», producteur Chancel, Jacques et Fauquet, Marie Hélène, 23 juin 1989, (54mn59s).
} 